\title{
Caracterização da matéria-prima com tamanho de partículas nanométricas utilizadas no processamento do compósito WC-10\%Co
}

\author{
Characterization of the raw material with \\ nanometric particle size used in the processing of \\ the composite WC-10\% Co
}

Adriano Corrêa Batista ${ }^{1}$, Amanda Maia Aguiar ${ }^{2}$, Hellen Cristine Prata de Oliveira ${ }^{2}$, Paulo Santos Assis ${ }^{2}$

\author{
${ }^{1}$ Coordenadoria de Física, Instituto Federal de Educação, Ciência e Tecnologia de Minas Gerais- IFMG, Campus Ouro \\ Preto, CEP: 35400-000, Ouro Preto, MG, Brasil. \\ ${ }^{2}$ Rede Temática de Engenharia de Materiais, Universidade Federal de Ouro Preto, CEP: 35400-000, Ouro Preto, MG, \\ Brasil. \\ e-mail: adrianocorrea77@gmail.com, amandamaia.ufop@gmail.com, hcpratamg@gmail.com, assis.ufop@gmail.com.
}

\begin{abstract}
RESUMO
O compósito WC-10\%Co também conhecido como Metal Duro de Carbeto de Tungstênico (WC) com Cobalto (Co) é um dos materiais mais produzidas pela indústria fabricante de metal duro, entretanto busca-se o melhor entendimento sobre o efeito de parâmetros de processo sobre as suas características estruturais, especialmente no que diz respeito à formação de fases metaestáveis - fases $\eta$. Neste trabalho, usamos o método de Rietveld aliado a difração de raios X (DRX), a microscopia eletrônica de varredura (MEV) e o Brunauer - Emmett - Teller (BET) para investigar a estrutura das matérias-primas utilizadas na produção de metal duro. Os tamanhos das partículas assim como as morfologias identificadas para os pós de WC e Co são reflexos do seu processo de fabricação que, introduzem tensões residuais para o pó de WC, a baixa cristalinidade e significante parcela de amorfo para o pó de Co. Estas características foram identificadas com a difração de raios X em comparação com o padrão de difração obtido e os bancos de dados cristalográficos. Após o processo de mistura dos pós foram quantificadas as fases e identificados os efeitos desse processo sobre as células unitárias de cada fase. Este trabalho mostra que a rota de caracterização empregada, com o uso do método de Rietveld, é eficiente para analisar a estrutura desses materiais durante seu processamento. Foi possível observar que o processo de mistura utilizando equipamento de moagem de alta energia promove alteração na densidade das células unitárias do WC “empurrando" átomos de Co para dentro de sua estrutura. Isso pode vir a acelerar o processo de dissolução de grande parte das partículas de WC durante a sinterização, contribuindo para a formação acentuada das fases metaestáveis eta - $\eta$, prejudicando o equilíbrio entre as propriedades mecânicas de dureza e tenacidade à fratura tornando a ferramenta ou peça muito dura e pouco tenaz.
\end{abstract}

Palavras-chave: WC nanométrico; metal duro; difração de raios X; caracterização de materiais; Método Rietveld.

\begin{abstract}
The composite WC- $10 \%$ Co is one of the materials most produced by the hardmetal fabrication industry. However, a better understanding of the effect of process parameters on their structural characteristics is sought, especially with respect to the formation of metastable phases - $\eta$. In this work, we used the Rietveld Method with X - Ray Diffraction (XRD), Scanning Electron Microscopy (SEM) and Brunauer - Emmett - Teller (BET) to investigate the structure of the raw materials used in the production of hardmetal. The particle sizes as well as the identified morphologies for the WC and Co powders are reflections of their manufacturing process, which introduce residual stresses for WC powder, low crystallinity and a significant amorphous portion for Co powder. These characteristics were identified with XRD in comparison to the obtained diffraction pattern and crystallographic databases. After the powder mixing process, the phases were quantified and the effects of this process were identified on the unit cells of each phase. This work shows that the characterization route using the Rietveld Method is efficient to analyze the structure of these materials during their processing. It was observed that the
\end{abstract}


mixing process using high energy milling equipment promotes a change in the density of WC unit cells by "pushing" Co atoms into their structure. This may accelerate the dissolution process of most WC particles during sintering. And contribute to the accentuated formation of metastable phases eta $-\eta$, damaging the balance between the mechanical properties of hardness and fracture toughness making the tool or piece very hard and not very tenacious.

Keywords: nanometric WC; hardmetal; X-ray diffraction; materials characterization; Rietveld method.

\section{INTRODUÇÃO}

O metal duro é a classe de materiais produzidos pela metalurgia do pó que apresenta a maior resistência ao desgaste e à dureza. É constituído por pelo menos um composto duro e uma matriz, que funciona como ligante. O composto duro é comumente o carbeto de tungstênio (WC), enquanto o ligante mais comum usado é o cobalto (Co), que apresenta as melhores características, ou seja, o metal duro é constituído de grãos de WC cementados pelo ligante rico em Co [1, 2]. A presença do ligante tem duas finalidades, melhorar o processo de sinterização pela presença de uma fase líquida e aumentar a tenacidade do produto final [3].

O metal duro é obtido durante o processo de sinterização em fase líquida, no qual ocorre a sinterização do pó de Co em fase líquida com o pó de WC à uma temperatura aproximada de $1400^{\circ} \mathrm{C}$. O Co difunde na estrutura contribuindo para a densidade e homogeneidade desejadas [4]. O Co é frequentemente usado como ligante na produção de metal duro devido à fácil molhabilidade do WC pelo Co líquido na temperatura de sinterização, resultando em aglomerados que contribuem para a densificação do metal duro $[5,6]$. Como todos os compósitos, as propriedades do metal duro dependem da combinação das propriedades de seus constituintes, WC e Co, e estes podem ser variados para obter as características desejadas $[7,8]$.

Os compósitos contendo os nanomateriais de WC e Co possuem excelente resistência à altas temperaturas e boa resistência à corrosão, sendo quimicamente e termicamente estáveis mesmo em altas temperaturas e também possuem alta dureza, boa resistência ao desgaste, boa resistência à fratura e possuem um alto valor de módulo de Young [4, 9, 11].

A caracterização a nível atômico destas matérias-primas, no que se refere à densidade, distribuição atômica, volume, dimensões da célula unitária e morfologia, contribui para o controle dos processos de fabricação. Além disso, é a base para identificação de fases, estrutura, distribuição de estabilizadores, porosidades e avaliação de desempenho de materiais [12, 13].

O interesse no metal duro com o tamanho de grão nanométrico deriva de um entendimento de que há um aumento generalizado na dureza com uma diminuição no tamanho do grão de metal duro tungstênio [4, 7, 8, 10]. Além disso, diferentes temperaturas durante o processo de sinterização também podem influenciar nos tamanhos dos grãos. Segundo Kumar et al. [1], o aumento da temperatura altera ou aumenta o formato dos grãos e diminui a porosidade do material.

Em 2016, Javanbakht, M. et al. [13] realizou um estudo com difração de raios X e Método de Rietveld com o objetivo de analisar as características estruturais e mecânicas dos aços inoxidáveis na metalurgia do pó. A difração de raios X aliada ao método de Rietveld são técnicas utilizadas na caracterização de materiais, possibilitando a análise qualitativa e quantitativa de fases, determinação da cristalinidade, parâmetros da rede cristalina, densidade e volume de célula unitária e medidas mais específicas, como tensão residual e textura [13,17].

O estudo desses materiais e compósitos é relevante pelo fato dos mesmos serem utilizados em inúmeros seguimentos da indústria de ferramentas de corte para usinagem, mineração, construção civil, brocas e minibrocas, suportes, motores elétricos, componentes de equipamentos da indústria de exploração e produção de óleo e gás, também é largamente utilizado na indústria automobilística [18].

Contudo, as ferramentas de corte à base de metal duro, ainda são, em grande parte, importadas, ressaltando a relevância do desenvolvimento tecnológico destas ferramentas no país. Estes materiais oferecem propriedades mecânicas tais como dureza e tenacidade à fratura que podem ser obtidas e melhoradas em função, sobretudo, dos seus aspectos microestruturais, sendo de importância o estudo e compreensão da formação de suas microestruturas.

A quantificação das fases é um passo fundamental na determinação da estrutura, propriedades e aplicações do material [14]. Sendo assim, o presente trabalho tem por finalidade caracterizar as matérias-primas utilizadas no processamento de carbetos cementados, o compósito metal duro - WC-10\%Co com pó de WC com tamanho de partícula nanométrica, obtendo a morfologia e tamanho de partículas através das técnicas de BET e MEV e identificação das fases, parâmetros estruturais, densidade, volume de célula unitária e a quantificação das fases presentes através do Método de Ritveld. 


\section{MATERIAIS E MÉTODOS}

As matérias-primas utilizadas neste trabalho foram o pó nanométrico de WC, com tamanho médio de partículas de $125 \mathrm{~nm}$ e o pó de Co, com tamanho médio de partícula de $3.6 \mu \mathrm{m}$. As Tabelas 1e 2 a seguir, mostram as análises químicas dos pós, fornecidas pelos fabricantes.

Tabela 1: Composição química do pó de WC nanométrico (\% em peso).

\begin{tabular}{c|c|c|c|c|c|c|c|c|c|c|c|c}
\hline ELEMENTO & $\mathbf{C}_{\text {Total }}$ & $\mathbf{C}_{\text {Livre }}$ & $\mathbf{O}$ & $\mathbf{A l}$ & $\mathbf{C a}$ & $\mathbf{C r}$ & $\mathbf{F e}$ & $\mathbf{M o}$ & $\mathbf{N i}$ & $\mathbf{S i}$ & $\mathbf{S n}$ & $\mathbf{W}$ \\
\hline QUANTIDADE & 6,18 & 0,14 & 0,10 & 2 & 2 & 10 & 35 & 12 & 4 & $<5$ & $<2$ & \multirow{2}{*}{ Base } \\
\hline UNIDADE & $\%$ & $\%$ & $\%$ & $\mathrm{ppm}$ & $\mathrm{ppm}$ & $\mathrm{ppm}$ & $\mathrm{ppm}$ & $\mathrm{ppm}$ & $\mathrm{ppm}$ & $\mathrm{ppm}$ & $\mathrm{ppm}$ & \\
\hline
\end{tabular}

Tabela 2: Composição química do pó de Co (\% em peso)

\begin{tabular}{c|c|c|c|c|c|c|c|c|c|c|c|c}
\hline ELEMENTO & $\mathbf{O}_{\mathbf{2}}$ & $\mathbf{C}$ & $\mathbf{S}$ & $\mathbf{N i}$ & $\mathbf{F e}$ & $\mathbf{C u}$ & $\mathbf{Z n}$ & $\mathbf{M n}$ & $\mathbf{N a}$ & $\mathbf{M g}$ & $\mathbf{C a}$ & $\mathbf{C o}$ \\
\hline QUANTIDADE & 0,55 & 1500 & 30 & 500 & $<10$ & $<20$ & $<20$ & $<10$ & $<10$ & $<10$ & $<10$ & \multirow{2}{*}{ Base } \\
\hline UNIDADE & $\%$ & $\mathrm{ppm}$ & $\mathrm{ppm}$ & $\mathrm{ppm}$ & $\mathrm{ppm}$ & $\mathrm{ppm}$ & $\mathrm{ppm}$ & $\mathrm{ppm}$ & $\mathrm{ppm}$ & $\mathrm{ppm}$ & $\mathrm{ppm}$ & \\
\hline
\end{tabular}

A mistura dos pós de WC e Co, segundo a composição WC-10\%Co (\%peso), foi realizada à seco por meio da moagem de alta energia com o moinho do tipo SPEX 8000 durante 40 minutos. A relação entre a quantidade de massa dos pós e esferas dentro do reservatório foi de 3:1, até preencher aproximadamente $25 \%$ do reservatório visando promover a homogeneização adequada.

O reservatório do moinho utilizado foi de metal duro não oferecendo risco de contaminação. Para diminuir o atrito entre a pulsão e as paredes da matriz e melhorar a aglomeração das partículas dos pós permitindo o manuseio dos corpos verde, foram utilizadas pastilhas de cânfora $\left(\mathrm{C}_{10} \mathrm{H}_{16} \mathrm{O}\right)$, substância cerosa, branca, pouco solúvel em água, que se dissolve facilmente no álcool, éter e demais solventes orgânicos e volatiliza-se a temperatura ambiente.

Todo o procedimento experimental foi realizado nos laboratórios da Escola de Minas: Departamento de Engenharia Metalúrgica e de Materiais - DEMET localizado na Universidade Federal de Ouro Preto - UFOP.

As medidas de área específica superficial foram realizadas pelo método de adsorção física de gás desenvolvido por Brunauer, Emmett e Teller (BET). Esta técnica BET foi realizada por adsorção de nitrogênio após a desgaseificação das amostras [19-20] usando um aparelho de análise superficial modelo Gemini 2360, Micromeritics. Assumindo partículas soltas e esféricas, o tamanho médio de partícula BET $\left(\mathrm{d}_{\mathrm{BET}}\right)$ são estimados pela equação (1):

$$
D_{B E T}=\frac{6}{\rho A_{B E T}}
$$

sendo,

$\mathrm{D}_{\mathrm{BET}}$, o tamanho médio de partícula em $\mu \mathrm{m}$;

$\mathrm{A}_{\mathrm{BET}}$, área de superfície $\mathrm{BET}$ em $\mathrm{m}^{2} / \mathrm{g}$;

$\rho$, densidade teórica em $\mathrm{g} / \mathrm{cm}^{3}$.

Por meio da DRX as fases presentes nos pós de WC, Co e na mistura WC-10\%Co foram identificadas, com o equipamento da marca Shimadzu, modelo XRD 7000, utilizando a radiação de $\mathrm{Cu} \mathrm{K \alpha}$, voltagem de $40 \mathrm{KV}$, corrente de $30 \mathrm{~mA}$, com $2 \theta$ variando de 5 a $120^{\circ}$ com passo de $0,01^{\circ}$ e tempo de 3 s por ponto. Os difratogramas obtidos dos pós e da mistura foram comparados com arquivos CIF (Crystallographic Information File) presentes nas Bases de Estruturas Cristalinas (BDEC) [21]. O procedimento de análise para a comparação foi feito entre as posições $2 \theta$ dos picos, distâncias interplanares e a intensidade relativa de cada pico.

Com o MEV foram observados os aspectos microestruturais das amostras em pó e detalhes da morfologia. Foi utilizado o MEV da marca Shimadzu, modelo SSX-550, voltagem de $10 \mathrm{keV}$ e elétrons secundários (SE).

Para a análise quantitativa de fases pelo Método de Rietveld, foi utilizado o programa General Structure Analysis 
System - GSAS [22]. Os parâmetros de refinamento selecionados durante a quantificação das fases incluem os fatores de escala, a função polinomial que define a radiação de fundo, o deslocamento da amostra, os parâmetros de rede de cada fase, parâmetros que definem a largura e o perfil dos picos calculados, e funções de orientação preferencial e assimetria. A função de perfil Pseudo-Voigt - 4/GSAS foi escolhida para o refinamento das fases para todas as amostras estudadas neste trabalho, devido à sua potencialidade de modelar picos com as funções Lorentiziana e Gaussiana juntamente com funções de assimetria.

\section{RESULTADOS}

\subsection{BET}

A Tabela 3 apresenta a densidade teórica, os resultados da área superficial encontrada na análise BET e tamanho médio de partícula calculados através da equação (1).

Tabela 3: Densidade teórica, Área superficial BET e tamanho de médio de partículas dos pós de WC, Co e da mistura WC- $10 \%$ Co.

\begin{tabular}{c|c|c|c}
\hline AMOSTRA & $\begin{array}{c}\text { DENSIDADE } \\
\text { TEÓRICA }\left(\mathbf{g} / \mathbf{c m}^{3}\right)\end{array}$ & $\begin{array}{c}\text { ÁREA SUPERFICIAL } \\
\text { BET }\left(\mathbf{m}^{2} \mathbf{g}\right)\end{array}$ & $\begin{array}{c}\text { TAMANHO DE MÉDIO } \\
\text { DE PARTíCULAS }\end{array}$ \\
\hline $\mathrm{WC}$ & 15,70 & 3,052 & $125 \mathrm{~nm}$ \\
\hline $\mathrm{Co}$ & 8,9 & 0,187 & $3,6 \mu \mathrm{m}$ \\
\hline $\mathrm{WC}-10 \% \mathrm{Co}$ & 14,60 & 1,085 & $378 \mathrm{~nm}$ \\
\hline
\end{tabular}

Considerando os valores de densidade teórica e a área superficial BET apresentados na tabela para as respectivas amostras, os valores de tamanhos de partículas para o pó de WC e para o pó de Co encontrados de acordo com equação (1) é $125 \mathrm{~nm}$ e $3,6 \mu \mathrm{m}$.

No método BET é possível relacionar a área superficial obtida com o tamanho médio de partículas, pois quanto maior a área superficial obtida menores serão os tamanhos médios das partículas [23]. Tal afirmação é coerente com os resultados apresentados na Tabela 3 e que por fim, correspondem ao valor informado pelo fabricante para o tamanho médio de partículas, provando assim que os valores de área superficial BET encontrados experimentalmente são resultados com acurácia.

Para a mistura WC-10\%Co, o valor dos tamanhos das partículas é $378 \mathrm{~nm}$. Tal valor deve-se ao fato que durante o processo de moagem, o pó de Cobalto pode ter tido seu tamanho de partícula reduzidos e adicionalmente o pó de WC fino se distribuiu em torno das partículas de Co, aumentando assim seu tamanho final na mistura quando analisadas. Processo esse que foi especialmente benéfico para obter a liga não homogênea no processo de sinterização a seguir.

\subsection{MEV}

As morfologias dos pós de WC e Co preparadas no moinho de bolas são mostrados na Figura 1 com aumentos de 10000x e 5000x. 

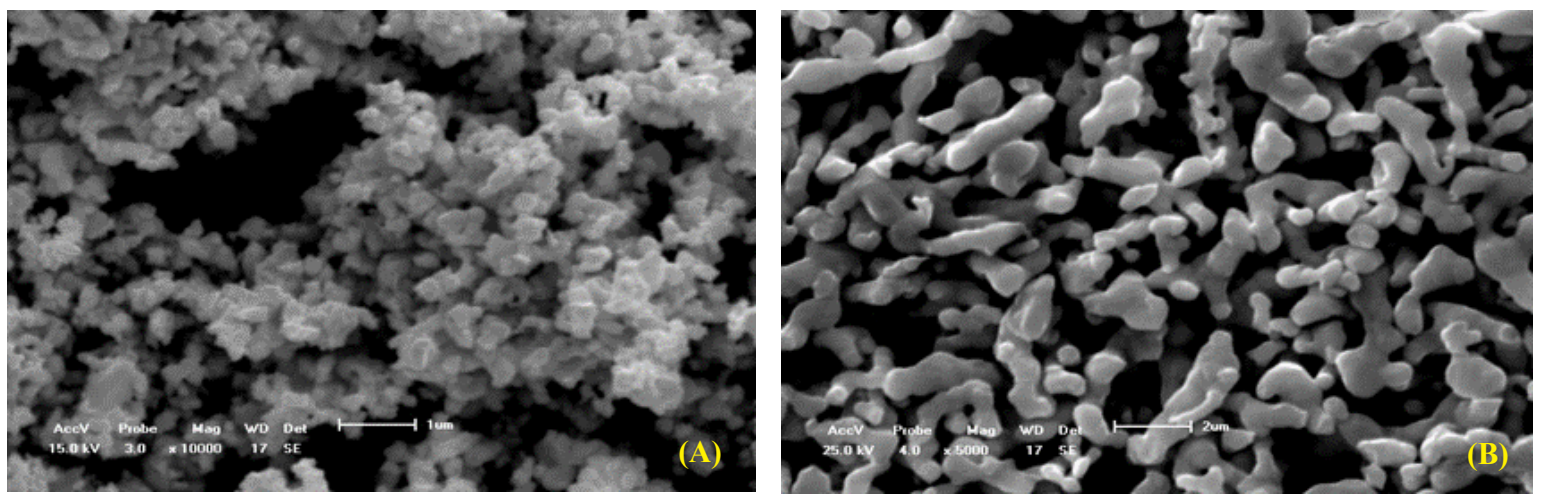

Figura 1: Micrografias dos pós de (A) WC e (B) Co, com aumento de 10000x e 5000x, respectivamente.

Foram verificados diferentes morfologias e tamanhos de partículas, sendo que as partículas do pó de WC nanométrico (A) apresentam morfologia arredondada, sendo menores e mais aglomeradas do que as partículas do pó de Co (B), que possui morfologia ligamental típica de processo de fabricação por atomização [3].

Estas imagens de MEV das amostras de WC e Co indicam que a granulometria está de acordo com a área específica das duas obtidas através do método BET de $2,911 \mathrm{~m}^{2} / \mathrm{g}$ da amostra $\mathrm{WC}$ e de $0,187 \mathrm{~m}^{2} / \mathrm{g}$ da amostra Co, pois quanto maior a área superficial obtida menores serão os tamanhos médios das partículas [23].

\subsection{DRX}

Com a análise qualitativa do padrão de difração de raios X deste material identificamos a fase WC hexagonal primitiva (hp) de acordo com arquivo CIF 9013303 da BDEC/Crystmet [21], como pode ser visto na Figura 2.

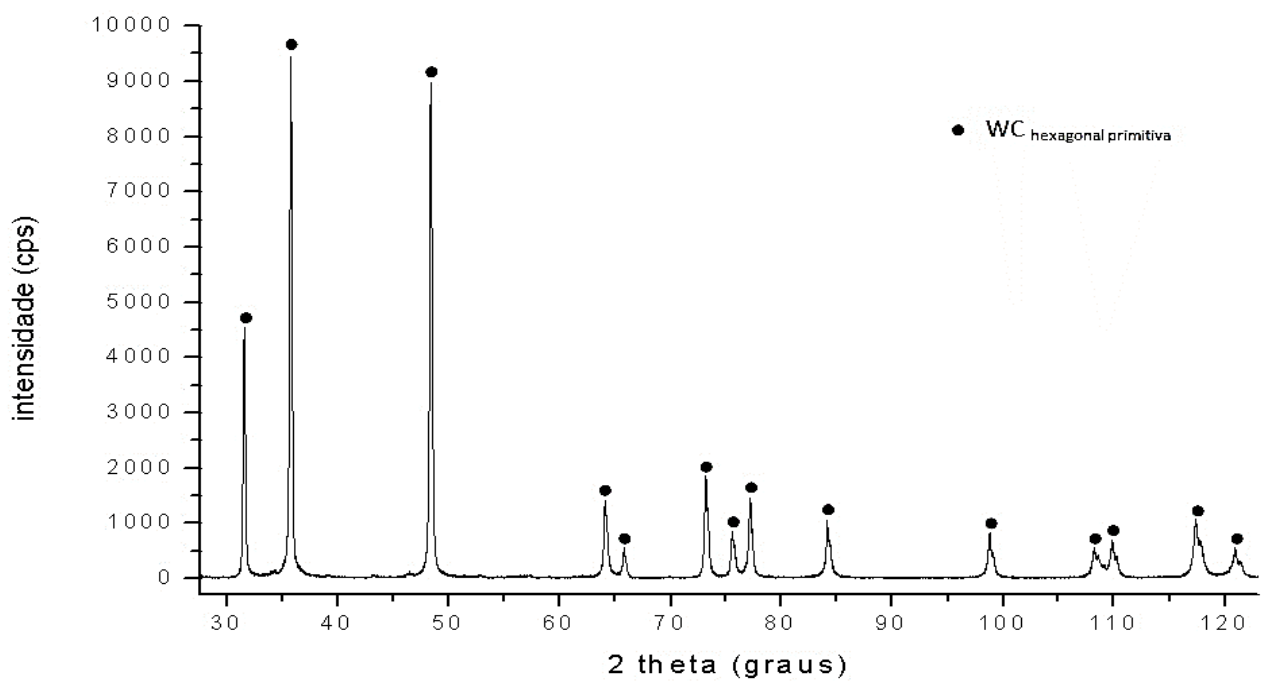

Figura 2: Difratograma do pó WC nanométrico [21].

O difratograma do pó nanométrico de WC mostrado na Figura 2, apresenta o background pouco ruidoso com a fase WC bem definida, picos pouco alargados indicando boa cristalinidade. Após revisão do banco de dados cristalográfico, observa-se que o comportamento estrutural deste pó é bem definido com pequenas variações toleráveis nas posições dos picos e distâncias interplanares, sendo que os picos deste difratograma estão em acordo e representam a formação da fase WC hexagonal primitivo (hp).

Quando em sua forma metálica, o tungstênio possui uma estrutura cristalina cúbica de corpo centrado, que é a mais estável. No entanto, para a formação do pó de WC um carbono é inserido na estrutura de tungstênio, o que resulta em uma nova estrutura cristalina, hexagonal primitiva [24]. Esta nova estrutura cristalina visa minimizar a energia interna do sistema. 
O difratograma do pó de Co da Figura 3 apresenta o background ruidoso indicando considerável fração de amorfo e picos alargados com baixa intensidade, configuração esta oriunda do processo de fabricação do pó.

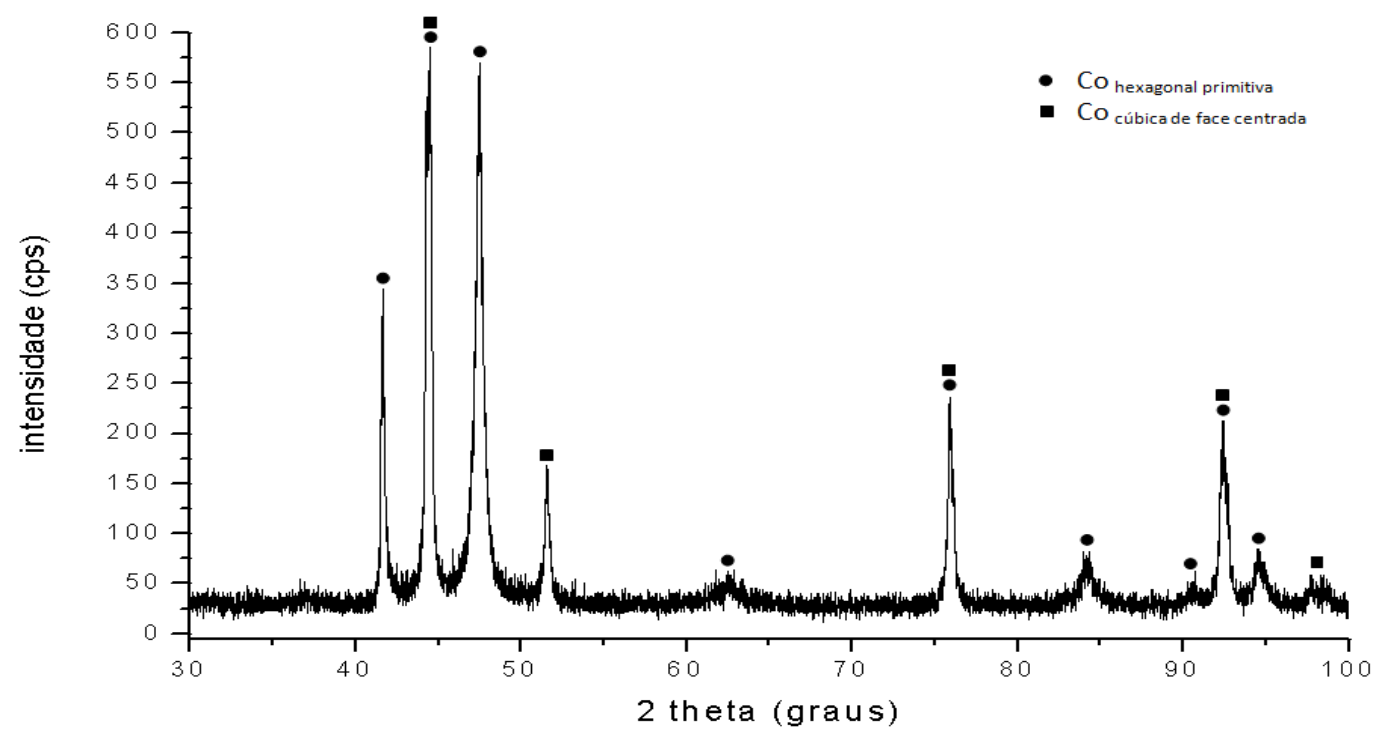

Figura 3: Difratograma do pó de Co - referências do banco de dados cristalográfico, Co (9010968/cfc) e Co (9008492/ hp) $[21]$.

Observa-se que o comportamento estrutural deste pó, não é bem definido possuindo duas fases, uma cubica de face centrada (cfc) e outra hp. O pico com a posição $51.56^{\circ}$ manifesta-se na estrutura do Co (cfc). Os demais picos com intensidades abaixo 84 (cps) representam o estágio de formação da fase Co (hp).

A Figura 4 mostra o difratograma dos pós de WC-10\%Co. Este revela a fase WC (hp) bem definida, apresentando picos pouco alargados e com consideráveis intensidades.

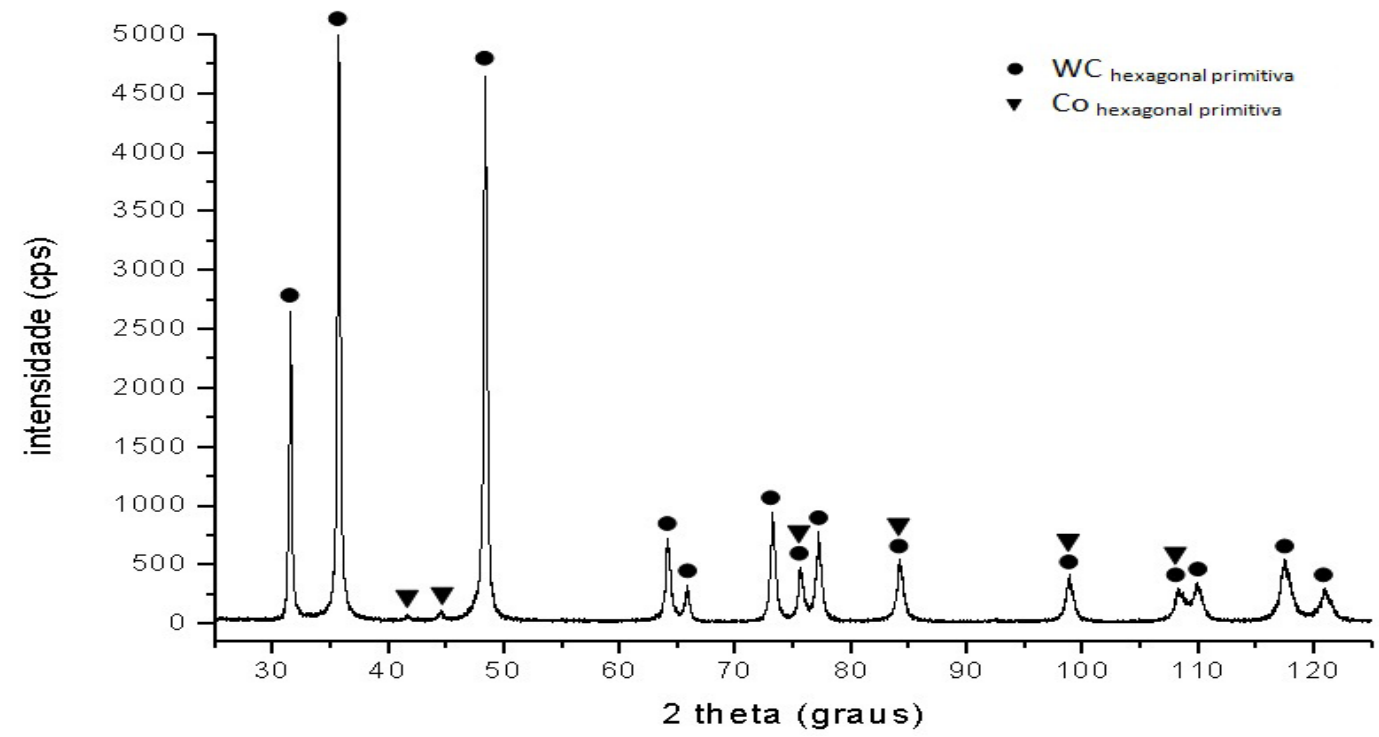

Figura 4: Difratograma da mistura WC-10\%Co - referências do banco de dados cristalográfico, WC (9013303/hp), Co (9008492/hp) [21].

Os picos típicos da fase Co (hp) também foram apresentados no difratograma da mistura $\mathrm{WC}-10 \% \mathrm{Co}$, contribuindo com leve alargamento dos picos de WC devido a sobreposição dos mesmos no intervalo de $46^{\circ}$ a $85^{\circ}$. Isso indica que Co foi incorporado com sucesso no pó de WC durante a mistura no moinho de bolas, sua contribuição para o difratograma é atenuada devido a sua representatividade neste pó compósito, com a quantidade de $10 \%$ em peso. 
A revisão do banco de dados cristalográfico indica a sobreposição de picos de $\mathrm{WC}$ e Co no intervalo de $74^{\circ}$ a $109^{\circ}$, gerando alargamento e pequenas diferenças nas posições dos mesmos quando comparados as fases individuais de WC e Co. O procedimento de mistura dos pós de WC e Co realizado com o moinho de esferas de alta energia foi utilizado com a intenção apenas de misturar os pós. Mas com a energia das colisões, possivelmente foi promovido o rearranjo atômico da fase Co (cfc) e da fração de amorfo do pó de Co para a fase Co (hp), também pode ter ocorrido a difusão dos átomos de Co na estrutura do WC.

\subsection{Método de Rietveld}

Foram utilizados os dados oriundos da difração de raios X obtidos das amostras dos pós de WC, Co, da mistura dos pós de WC-10\%Co, objetivando a determinação da estrutura, densidade e volume de célula unitária e quantificação das fases cristalinas presentes com o Método de Rietveld.

A minimização por mínimos quadrados gera os parâmetros necessários para a avaliação do refinamento. Um bom ajuste depende do quão adequado é o modelo, ou seja, se o modelo contém os parâmetros necessários para descrever a estrutura cristalina, bem como do alcance do mínimo global e não local.

As amostras analisadas neste trabalho foram em forma de pó, estruturas distintas e que apresentam características que as diferem, como o processo de fabricação, tamanho de grão e população estatística, disposição de fases presentes e fração de amorfo, variáveis que interferem e diferem o refinamento para cada tipo de material.

Na sequência, são apresentadas as informações obtidas com o refinamento das fases com informações que descrevem toda a estrutura das fases estudadas. Os parâmetros de rede das fases foram fornecidos ao final do refinamento junto com os valores de volume e densidade da célula unitária e os valores da quantificação das fases presentes para todas as amostras.

A Tabela 4 contém informações obtidas com o método de Rietveld que descrevem toda a estrutura da fase de WC referente ao pó com tamanho de partícula nanométrica.

Tabela 4: Parâmetros de rede e índices de refinamento do pó de WC nanométrico.

\begin{tabular}{c|c|c}
\hline \multicolumn{3}{c}{ FASE WC - GRUPO ESPACIAL Pm2 (187) - hp } \\
\hline $\mathrm{a}(\AA)$ & $\mathrm{b}(\AA)$ & $\mathrm{c}(\AA)$ \\
\hline $2,90511(2)$ & $2,90511(2)$ & $2,83873(3)$ \\
\hline $\mathrm{x}(\mathrm{W})$ & $\mathrm{y}(\mathrm{W})$ & $\mathrm{z}(\mathrm{W})$ \\
\hline $0,0000(0)$ & $0,0000(0)$ & $0,0000(0)$ \\
\hline $\mathrm{x}(\mathrm{C})$ & $\mathrm{y}(\mathrm{C})$ & $\mathrm{z}(\mathrm{C})$ \\
\hline $0,6667(0)$ & $0,3333(0)$ & $0,5000(0)$ \\
\hline Volume $\left(\AA^{3}\right)$ & Densidade $\left(\mathrm{g} / \mathrm{cm}^{3}\right)$ & 100 \\
\hline $20,748(0)$ & 16,523 & Fase $(\%)$ \\
\hline
\end{tabular}

a-WC ou somente WC é um carboneto de contém partes iguais de átomos de tungstênio e carbono (50/50) e possui rede cristalina primitiva hexagonal (única estrutura estável) com as constantes de célula unitária a $=2,906 \AA$ e c $=2,837 \AA$ $[24,28]$. Comparando estes com os resultados do método de Rietveld apresentados na Tabela 4 , valores dos parâmetros a = $2,905 \AA$ e c $=2,839 \AA$ A observa-se que estes se encontram muito próximos. Além disso, valores de posição atômica, densidade, volume de célula unitária e quantidade de fase na estrutura cristalina são apresentados. No caso do pó de WC, 100\% da estrutura cristalina é composta por WC hexagonal primitiva como pode ser visto na Tabela 4.

Na tabela 5 são mostrados os parâmetros de rede e índices de refinamento do pó de Co. Em acordo com a base de dados Crystmet, foram identificadas duas fases para o Co em pó, cúbica de face centrada (cfc) e hexagonais compacta (hc). A rede cristalina he possui valores de células unitária $a=2,5071 \AA, b=2,5071 \AA$ e $c=4,0695 \AA[17,25]$ e cfc possui os valores de célula unitária $\mathrm{a}=\mathrm{b}=\mathrm{c}=3,5447 \AA[26,27,29]$. Os resultados do Método de Rietveld apresentados na Tabela 5, mostra os valores dos parâmetros da rede cristalina he e cfc para o Co e sua proximidade com os valores encontrados na literatura, provando assim a qualidade do refinamento. 
Tabela 5: Parâmetros de rede e índices de refinamento do pó de Co.

\begin{tabular}{c|c|c}
\hline \multicolumn{3}{c}{ FASE Co - GRUPO ESPACIAL P63/mmc (194) - hc } \\
\hline $\mathrm{a}(\AA)$ & $\mathrm{b}(\AA)$ & $\mathrm{c}(\AA)$ \\
\hline $2.5082(2)$ & $2.5082(2)$ & $4.0742(3)$ \\
\hline $\mathrm{x}(\mathrm{Co})$ & $\mathrm{y}(\mathrm{Co})$ & $\mathrm{z}(\mathrm{Co})$ \\
\hline $0.3333(0)$ & $0.6667(0)$ & $0.2500(0)$ \\
\hline Volume $\left(\AA^{3}\right)$ & Densidade $\left(\mathrm{g} / \mathrm{cm}^{3}\right)$ & Fase $(\%)$ \\
\hline $22.197(4)$ & 8.819 & $75.6(5)$ \\
\hline \multicolumn{3}{c|}{ FASE Co - GRUPO ESPACIAL Fmm (225) - cfc } \\
\hline $3.5445(3)$ & $\mathrm{b}(\AA)$ & $\mathrm{c}(\AA)$ \\
\hline $\mathrm{x}(\mathrm{Co})$ & $3.5445(3)$ & $\mathrm{z}(\mathrm{Co})$ \\
\hline $0.0000(0)$ & $\mathrm{y}(\mathrm{Co})$ & $0.0000(0)$ \\
\hline Volume $\left(\AA^{3}\right)$ & $0.0000(0)$ & Fase $(\%)$ \\
\hline $44.531(9)$ & Densidade $\left(\mathrm{g} / \mathrm{cm}^{3}\right)$ & $24.4(6)$ \\
\hline
\end{tabular}

Além disso, na Tabela 5 é possível também observar a quantidade de fase na estrutura cristalina. No caso do pó de Co, o refinamento dos dados do padrão de raios X para o Co revelou a presença de duas fases, uma do Co (hp) com 75.58 $\%$ e da fase de Co (cfc) com $24.41 \%$, da constituição cristalina da amostra.

Na tabela 6 são mostrados os parâmetros de rede e índices de refinamento da mistura dos pós de WC e Co. Os parâmetros de rede das duas fases foram obtidos com o refinamento dos dados do padrão de difração de raios X, obtendo os valores de volume e densidade da célula unitária e os valores da quantificação das fases de WC (hp) e Co (hp).

Tabela 6: Parâmetros de rede e índices de refinamento da mistura dos pós de WC e Co.

\begin{tabular}{|c|c|c|}
\hline \multicolumn{3}{|c|}{ FASE WC - GRUPO ESPACIAL Pm2 (187) - hp } \\
\hline $\mathrm{a}(\AA)$ & $\mathrm{b}(\AA)$ & $\mathrm{c}(\AA)$ \\
\hline $2.9056(6)$ & $2.9056(6)$ & $2.8393(3)$ \\
\hline $\mathrm{x}(\mathrm{W})$ & $\mathrm{y}(\mathrm{W})$ & $\mathrm{z}(\mathrm{W})$ \\
\hline $0.0000(0)$ & $0.0000(0)$ & $0.0000(0)$ \\
\hline $\mathrm{x}(\mathrm{C})$ & $\mathrm{y}(\mathrm{C})$ & $\mathrm{z}(\mathrm{C})$ \\
\hline 0.6667 & 0.3333 & 0.5000 \\
\hline Volume $\left(\AA^{3}\right)$ & Densidade $\left(\mathrm{g} / \mathrm{cm}^{3}\right)$ & Fase $(\%)$ \\
\hline 20.71 & 20.36 & 90.51 \\
\hline \multicolumn{3}{|c|}{ FASE Co - GRUPO ESPACIAL P63/mmc (194) - hc } \\
\hline $\mathrm{a}(\AA)$ & $\mathrm{b}(\AA)$ & $\mathrm{c}(\AA)$ \\
\hline 2.5458 & 2.5458 & 4.1464 \\
\hline $\mathrm{x}(\mathrm{Co})$ & $\mathrm{y}(\mathrm{Co})$ & $\mathrm{z}(\mathrm{Co})$ \\
\hline 0.3333 & 0.6667 & 0.2500 \\
\hline Volume $\left(\AA^{3}\right)$ & Densidade $\left(\mathrm{g} / \mathrm{cm}^{3}\right)$ & Fase $(\%)$ \\
\hline 23.27 & 10.01 & 9.49 \\
\hline
\end{tabular}

A fase WC (hp) teve sua quantidade indicada pelo refinamento em $90.51 \%$ e a quantidade da fase Co (hc) em 9,49 $\%$. A fase de Co ( $\mathrm{cfc}$ ) não foi aceita pelo refinamento gerando fortes divergências, impossibilitando o mesmo, indicando a ausência desta fase na composição deste material.

Os picos da fase Co manifestam-se em sua grande maioria em sobreposição aos picos de WC, causando leve alargamento dos mesmos e uma pequena parcela dos picos junto ao background devido às quantidades de cada fase presentes na amostra, 90.50 \% de WC e 9.49 \% de Co. A fase de Co (cfc) não foi aceita pelo refinamento, gerando fortes divergências, 
impossibilitando o uso do mesmo, indicando que esta fase não existe neste material homogeneizado obtido pela moagem de alta energia ou está abaixo do limite de detecção da técnica (comparar Fig. 3 com Fig. 4).

Com o auxílio da Tabela 6 observa-se os parâmetros de rede das duas fases obtidas com o refinamento, como os valores de volume e densidade da célula unitária. Com as informações obtidas no refinamento do padrão de difração de raios $\mathrm{X}$ do pó de WC e da mistura com o Co é observado aumento da densidade de célula unitária do WC presente na mistura, passando de $16,52 \mathrm{~g} / \mathrm{cm}^{3}$ para $20,35 \mathrm{~g} / \mathrm{cm}^{3}$. Os parâmetros de rede $a, b$ e $c$ mostram baixa variação nos valores de volume como pode ser visto na Tabela 7.

Tabela 7: Parâmetros de rede, volume e densidade de célula unitária do pó de WC e após sua mistura com Co.

\begin{tabular}{c|c|c|c}
\hline \multicolumn{2}{c|}{ PÓ WC } & \multicolumn{2}{c}{ MISTURA WC-10\%CO } \\
\hline $\mathrm{a}(\AA)$ & $2.9051(1)$ & $\mathrm{a}(\AA)$ & $2.905(6)$ \\
\hline $\mathrm{b}(\AA)$ & $2.9051(1)$ & $\mathrm{b}(\AA)$ & $2.905(6)$ \\
\hline $\mathrm{c}(\AA)$ & $2.8387(2)$ & $\mathrm{c}(\AA)$ & $2.839(3)$ \\
\hline Volume $\left(\AA^{3}\right)$ & $20.74(8)$ & Volume $\left(\AA^{3}\right)$ & $20.71(4)$ \\
\hline Densidade $\left(\mathrm{g} / \mathrm{cm}^{3}\right)$ & $16.52(3)$ & Densidade $\left(\mathrm{g} / \mathrm{cm}^{3}\right)$ & $20.35(9)$ \\
\hline
\end{tabular}

Analisando os dados da Tabela 7 pode-se obter o valor da massa total de átomos na célula unitária do pó de WC. Considerando as quantidades de átomos de $\mathrm{W}$ e $\mathrm{C}$ mais um átomo de Co por célula unitária, obtemos o valor de densidade da célula unitária do pó de WC na mistura. Valor este obtido com método de Rietveld para a densidade de $20,35 \mathrm{~g} / \mathrm{cm}^{3}$.

\section{CONCLUSÕES}

As micrografias dos pós de WC e Co revelaram diferentes morfologias e tamanhos de partículas, sendo que os pós de WC nanométricos apresentaram partículas menores e mais aglomeradas do que os pós de Co.

As Tabelas de composição química dos pós apresentam baixa presença de elementos contaminantes incapazes de formar estruturas, fases nas quais fossem possíveis de se identificar com a difração de raios X.

$\mathrm{O}$ uso da análise qualitativa por difração de raios X das fases para as amostras em pó do WC, Co e da mistura dos pós de WC-10\%Co mostram os efeitos do processo de fabricação para o pó de Co, sendo identificadas as fases de cúbica primitiva e hexagonal primitiva, em meio a fração de amorfo.

O uso do Método de Rietveld revelou as quantidades das fases cristalinas presentes em cada amostra. O refinamento dos dados oriundos da difração de raios X para o pó de WC indicou pelos índices de qualidade do refinamento, reflexos do processo de fabricação como a carburetação e a moagem de alta energia utilizada para reduzir o tamanho de grão, introduzindo forte tenção mecânica nesta estrutura.

O refinamento do pó de Co revelou $75.58 \%$ da presença da fase hp e $24.41 \%$ da fase cfc. No difratograma nota-se a presença da fração amorfa, tornando o background relativamente ruidoso.

A análise quantitativa de fases para a mistura WC-10\%Co revelou $90.50 \%$ de presença da fase WC (hp) e $9.49 \%$ da fase de Co (hp). Valores aproximados da relação teórica de $90 \%$ de WC e $10 \%$ de Co em peso.

O Método de Rietveld se mostrou uma ferramenta capaz de obter dados precisos, ampliando a eficiência da identificação das fases, quantificação e modelagem das mesmas. Com estas informações obtemos toda a "identidade" estrutural dos pós de partida com maior quantidade de informações, as quais permitirão o monitoramento da evolução destas estruturas com o processo de sinterização, o que poderá ser relacionado com as propriedades mecânicas e suas variações com o surgimento de novas fases como $\mathrm{Co}_{3} \mathrm{~W}_{3} \mathrm{C}$ e $\mathrm{Co}_{6} \mathrm{~W}_{6} \mathrm{C}$ e o seu comportamento na composição estrutural do metal duro.

\section{AGRADECIMENTOS}

A realização do presente trabalho só foi possível devido ao apoio financeiro do CNPq e CAPES. Agradecemos aos laboratórios do Departamento de Engenharia Metalúrgica e de Materiais - DEMET e o laboratório de Difração de Raios X do departamento de Física - DEFIS da Universidade Federal de Ouro Preto - UFOP. 


\section{BIBLIOGRAFIA}

[1] KUMAR, A., SINGH, K., \& PANDEY, O. P. "Sintering behavior of nanostructured WC-Co composite." Ceramics International, v. 37, n. 4, pp. 1415-1422, May 2011.

[2] ORTNER, H. M., ETTMAYER, P., KOLASKA, H., et al. "The history of the technological progress of hardmetals." International Journal of Refractory Metals and Hard Materials, n.49, pp. 3-8, 2015.

[3] GRUPO SETORIAL DE METALURGIA DO PÓ. “A Metalurgia do Pó: alternativa econômica com menor impacto ambiental.” Metallun Eventos Técnicos, São Paulo, 2009.

[4] FANG, Z., MAHESHWARI, P., WANG, X., et al."An experimental study of the sintering of nanocrystalline WC-Co powders." International Journal of Refractory Metals and Hard Materials, v. 23, n. 4-6, pp. 249-257, July-November 2005.

[5] PÖTSCHKE, J., SÄUBERLICH, T., VORNBERGER, A., et al. "Solid state sintered nanoscaled hardmetals and their properties." International Journal of Refractory Metals and Hard Materials, n. 72, pp. 45-50, 2018.

[6] GRILLI, M. L., BELLEZZE, T., GAMSJÄGER, E., et al., "Solutions for Critical Raw Materials under Extreme Conditions." A Review by Materials, v. 10, n. 3, p. 285, 2017.

[7] FANG, Z. Z., WANG, X., RYU, T., et al."Synthesis, sintering, and mechanical properties of nanocrystalline cemented tungsten carbide - a review." International Journal of Refractory Metals and Hard Materials, v. 27, n. 2, pp. 288-299, March 2009.

[8] FABIJANIĆ, T. A., ALAR, Z., ĆORIĆ, D., "Influence of consolidation process and sintering temperature on microstructure and mechanical properties of near nano and nanostructured WC-Co cemented carbides." International Journal of Refractory Metals and Hard Materials, n. 54, pp. 82-89, 2016.

[9] YANG, Q., YANG, J., YANG, H., NI, G., et al. "Synthesis of ultrafine WC-10Co composite powders with carbon boat added and densification by sinter-HIP." International Journal of Refractory Metals and Hard Materials, n. 62, pp. 104-109, 2017.

[10] TSAI, K. M., HSIEH, C. Y., \& LU, H. H., "Sintering of binderless tungsten carbide." Ceramics International, v. 36, n. 2, pp. 689-692, March 2010.

[11] KONYASHIN, I.; KLYACHKO, L. I., "History of cemented carbides in the Soviet Union." International Journal of Refractory Metals and Hard Materials, n. 49, p. 9-26, March 2015.

[12] WANG, D., LIU, W., FENG, Q., et al."Effect of inorganic/organic ratio and chemical coupling on the performance of porous silica/chitosan hybrid scaffolds.” Materials Science and Engineering: C, n. 70, pp. 969-975, 2017.

[13] JAVANBAKHT, M.; SALAHINEJAD, E.; HADIANFARD, M. J. "The effect of sintering temperature on the structure and mechanical properties of medical-grade powder metallurgy stainless steels." Powder technology, n. 289, pp. 37-43, February 2016.

[14] RIETVELD, H. M., “The rietveld method.” Physica Scripta, v. 89, n. 9, p. 098002, August 2014.

[15] ANDO, Y., OHISHI, Y., SUZUKI, K., et al. "Rietveld refinement of the crystal structure of perovskite solar cells using $\mathrm{CH}_{3} \mathrm{NH}_{3} \mathrm{PbI}_{3}$ and other compounds", In: AIP Conference Proceedings, v. 1929, n. 1, pp. 020003, January 2018.

[16] CLUFF, D. R., CORBIN, S. F., GHARGHOURI, M. A., "Rietveld refinement on in-situ neutron diffraction during sintering of NiTi from elemental powders", Journal of Alloys and Compounds, n. 732, pp. 845-855, January 2018.

[17] KHALILI S, SOLEIMANIAN V, MOKHTARI A., "Developing a method for the evaluation of dislocation parameters from the Rietveld refinement procedure", Powder Diffraction, v. 31, n. 3, pp. 198-204, 2016.

[18] KONYASHIN, I., RIES, B., HLAWATSCHEK, S., et al."Novel industrial hardmetals for mining, construction and wear applications." International Journal of Refractory Metals and Hard Materials, n. 71, pp. 357-365, 2018.

[19] BRUNAUER, S., EMMETT, P. H., TELLER, E., “Adsorption of gases in multimolecular layers", Journal of the American chemical society, v. 60, n. 2, pp. 309-319, February 1938.

[20] LASHEEN, M. R., EL-SHERIF, I. Y., TAWFIK, M. E., et al."Preparation and adsorption properties of nano magnetite chitosan films for heavy metal ions from aqueous solution", Materials Research Bulletin, n. 80, pp. 344-350, August 2016.

[21] BASES DE ESTRUTURAS CRISTALINAS. http://bdec.dotlib.com.br/cliente/login. Acessado em abril de 2018.

[22] LARSON, A. C., VON DREELE, R. B., "General Structure Analysis Systen (GSAS)”. Los Alamos: Los Alamos National Laboratory Report LAUR, pp.8 6-748, 2004. 
[23] MEL'GUNOV, M. S., AYUPOV, A. B. "Direct method for evaluation of BET adsorbed monolayer capacity". Microporous and Mesoporous Materials, n. 243, pp. 147-153, February 2017.

[24] KURLOV, A. S., GUSEV, A. I., Tungsten carbides: Structure, Properties and Application in Hardmetals, Springer Ser. Mater. Sci, n. 184, pp. 34-6, 2013.

[25] CHARI, A., Computational Thermodynamics of CoNiGa High Temperature Shape Memory Alloys, Tese de Doutorado, Texas A \& M University, Texas, 2012.

[26] YUAN, Y., FU, L., LI, J., “Annealing effect on the mechanical properties of ultrafine WC-Co materials.” Journal of Applied Research and Technology, n. 15, pp. 396-401, 2017.

[27] LI, Y. D., LI, L. Q., LIAO, H. W., et al."Preparation of pure nickel, cobalt, nickel-cobalt and nickel-copper alloys by hydrothermal reduction”, Journal of Materials Chemistry, v. 9, n. 10, pp. 2675-2677, 1999.

[28] BATISTA, A. C., OLIVEIRA, H.C.P., FILGUEIRA, M., et al."Effect of high-energy milling and sintering temperature on the structure of a hardmetal." Indian Journal of Applied Research, DOI: 10.13140/RG.2.2.19940.83840. n. 8, pp. 1-7, 2018.

[29] MANFRIDINI, A. P. A., GODOY, G. C. D., SANTOS, L. A., "Structural characterization of plasma nitrided interstitial-free steel at different temperatures by SEM, XRD and Rietveld method." Journal of Materials Research and Technology, n. 6, pp. 65-70, 2017.

\section{ORCID}

Hellen Cristine Prata de Oliveira

Adriano Corrêa Batista

Amanda Maia Aguiar

Paulo Santos Assis https://orcid.org/0000-0001-7390-6987

https://orcid.org/0000-0002-3515-7281

https://orcid.org/0000-0003-4511-002X

https://orcid.org/0000-0003-0874-4162 